\title{
Subintimal angioplasty and stenting in chronic total femoropopliteal artery occlusions: Early- and mid-term outcomes
}

\author{
Ersan Tatli ${ }^{1}$, Ali Buturak ${ }^{2}$, Osman Kayapınar ${ }^{1}$, Emir Dogan ${ }^{1}$, \\ Mustafa Alkan', Yasemin Gunduz ${ }^{3}$ \\ ${ }^{1}$ Department of Cardiology, Duzce University School of Medicine, Duzce, Turkey \\ ${ }^{2}$ Department of Cardiology, Acibadem University Hospital, Istanbul, Turkey \\ ${ }^{3}$ Department of Radiology, Sakarya University School of Medicine, Sakarya, Turkey
}

\begin{abstract}
Background: This study was conducted to evaluate the initial and mid-term patency rates of chronic total femoropopliteal artery (FPA) occlusions treated by subintimal angioplasty (SIA) and stenting.
\end{abstract}

Methods: From March 2010 to February 2013, 74 patients were included in the study. Seventy two patients with total occlusion of the FPA and good distal runoff (2 or 3 patent vessels) were treated with percutaneous SIA and stenting. All patients had severe claudication or critical limb ischemia. In all cases, the procedure was performed with a contralateral approach. Follow-up was done at 6 months with clinical evaluation and color-Doppler. If it was necessary, peripheric angiography was performed.

Results: Immediate technical success was achieved in 72 (97\%) patients. Two (3\%) distal embolizations, 2 (3\%) groin hematomas, 1 (1\%) femoral pseudoaneurysm and 1 (1\%) rupture of the junction-external iliac-superficial femoral artery occurred. All of the complications were treated successfully. Total occlusion in 1 patient and critical occlusion in 3 patients were showed at the $6^{\text {th }}$ month. Patency rate at the sixth month was $94 \%$ with a stent length of $13.4 \pm 8.2 \mathrm{~cm}$.

Conclusions: Percutaneous SIA and stenting for chronic total of the FPA occlusion showed good initial and mid-term patency rates, with few periprocedural complications. (Cardiol J 2015; 22, 1: 115-120)

Key words: total occlusion, femoropopliteal artery, subintimal angioplasty, stenting

\section{Introduction}

Endovascular treatment of occlusive peripheral arterial disease has been markedly increased in the last decade in cases of critical limb ischemia of lower extremity arteries or claudicants. Several techniques have been improved to achieve higher procedural success and patency rates especially in chronic total occlusions of superficial femoral or popliteal arteries since its first description by Bolia et al. in 1989 [1, 2].

Subintimal angioplasty (SIA) has been used as an acceptable and effective technique which allows recanalization of long diffuse chronic total

Address for correspondence: Dr Ersan Tatli, Department of Cardiology, Duzce University School of Medicine, Duzce, Turkey, tel: +90505 67890 99, e-mail: ersantatli@yahoo.com

Received: 02.03.2014 Accepted: 23.04.2014 
occlusions in superficial femoral and popliteal arteries. SIA has previously been used as as an alternative to conventional percutaneous transluminal angioplasty (PTA) when the latter technique fails. There is also a group of patients with critical lower limb ischemia and significant co-morbidities who are not eligible for surgical interventions. Revascularization with SIA offers reduced rates of amputations and improves wound healing in patients with critical limb ischemia (CLI) in addition to improvement in claudication status in this group of patients [3-6]. Although SIA has been accepted as a successful technique especially in long chronic femoropopliteal occlusions, low patency rates compared with surgical bypass (56-70\% at 1 year follow-up) remain the main criticism regarding its independent use [7-9]. However, revascularization by SIA leads to high limb salvage rates in patients with CLI indicating that reocclusion incidences did not exactly reflect clinical outcomes. Patency rates which are closely related to relief of symptoms in claudicants may also be improved by SIA, but in contrast to CLI patients, patency status of the artery determines the clinical success provided by the intervention.

The loss of the patency following intervention is multifactorial. Renal disease, diabetes mellitus, smoking, occlusion length and the number of distal runoff arteries are factors that adversely affect primary and long-term patency rates following SIA [10-12]. According to previous reports in the literature, high rate of occlusion occurs early during the first 6 months suggesting that the reocclusion may be related to elastic recoil with residual stenosis and/or flow limiting spiral dissection [13].

Based on these data, it may be suggested that the routine adjunction of stents to subintimal recanalization could provide improvement of patency and clinical outcomes by optimization of the SIA technique. We aimed to evaluate the clinical outcomes and mid-term patency rates following SIA and stenting of chronic occlusions in long femoropopliteal lesions.

\section{Methods}

\section{Study population}

The study was a prospective single center study including patients with either severe, lifestyle limiting intermittent claudication or CLI presenting with at least one chronic total occlusion of the superficial femoral artery (SFA) and/or popliteal artery with good distal runoff. The clinical severity of the claudication and limb ischemia was defined according to the Rutherford classification [14]. Between March 2010 to February 2013, 74 patients with angiographically documented chronic total occlusion in the femoropopliteal arteries (SFA plus popliteal) and at least a 6 -month history of intermittent claudication and/or CLI were included in the study. All of the participants' clinical status varied between Rutherford categories 1 to 6 which was previously defined [14]. Successful intraluminal wire passage through the lesion, history of severe contrast allergy or hypersensitivity, hypersensitivity to aspirin and/or clopidogrel, systemic coagulopathy or hypercoagulation disorders and acute limb ischemia were excluded. All of the patients gave written informed consent and the study was approved by the local Ethics Committee.

\section{Procedure}

All the patients were premedicated with dual anti-platelet therapy (aspirin $100 \mathrm{mg} /$ day plus clopidogrel $75 \mathrm{mg}$ /day) for 3 days before the procedure. After contralateral femoral puncture and insertion of a $7 \mathrm{~F}$ sheath, intra-arterial bolus of 5,000 IU heparin was injected to maintain an activated clotting time $>200 \mathrm{~s}$. SIA was performed as previously described by Bolia $[1,2]$. Entry transition to the subintimal space and re-entry to the intraluminal space was performed using a soft angled tip or j-tip hydrophilic 0.035-inch Raptor guidewires (InSitu, USA) with support of a $6.5 \mathrm{~F}$ Sheathless guide catheter (Asahi, Japan). The guidewire was advanced with a looped shape through the subintimal space. If the penetration was not achieved by the hydrophilic guidewire, stiffer and more steerable wires such as 0.018 inch Treasure $(12 \mathrm{~g}-180 / 300 \mathrm{~cm})$ or $0.014 / 0.018$ inch Astato $(20$ or $30 \mathrm{~g}-180 / 300 \mathrm{~cm}$ ) wires were used. After confirmation of successful distal re-entry, standard dilatation of the target lesion was performed using appropriately sized GliderfleX balloon catheter (TriReme Medical, USA). Then, self-expandable nitinol stents (Polaris, QualiMed, Germany) were implanted to cover the entire lesion. The stent size was determined to be 1 to $2 \mathrm{~mm}$ larger than the reference vessel diameter and subsequent post-dilatation of stents was performed to all cases (Figs. 1, 2). After completion of the procedure, all patients were prescribed to dual antiplatelet therapy with aspirin plus clopidogrel for 6 months and aspirin $100 \mathrm{mg} /$ day indefinitely after the $6^{\text {th }}$ month.

\section{Follow-up}

Patients were evaluated with physical examination and duplex scanning of the treated artery 


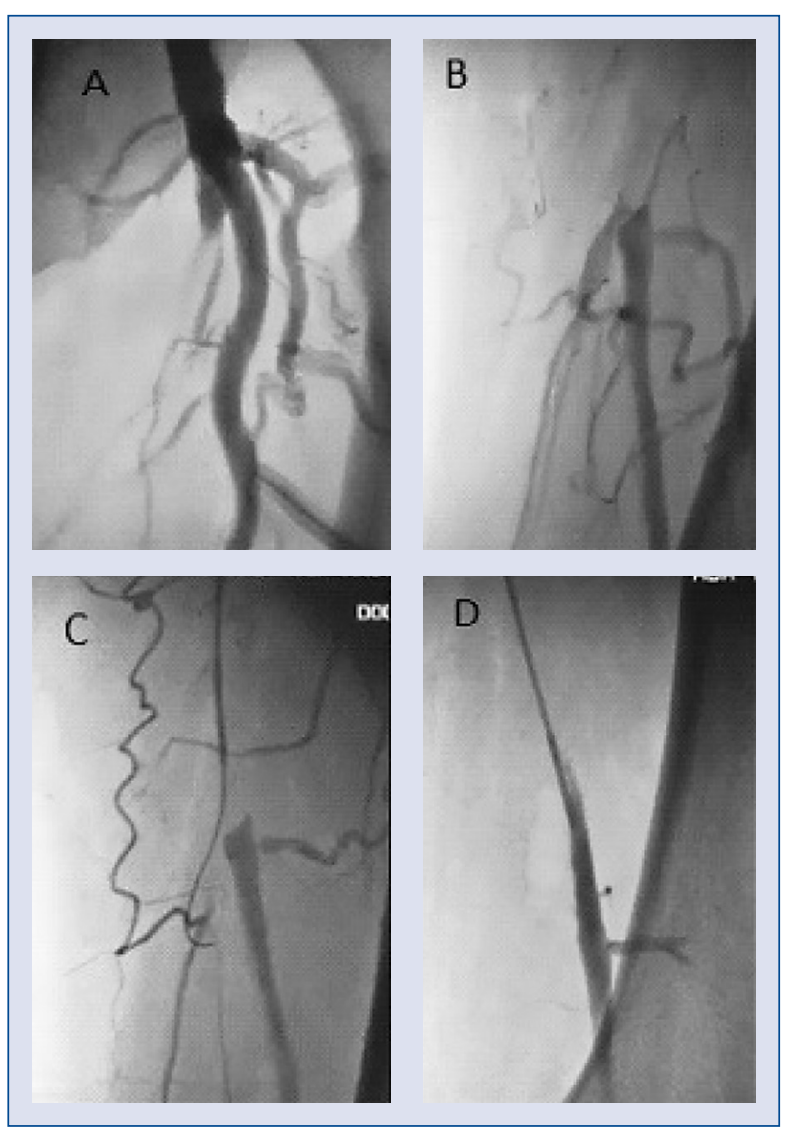

Figure 1. Subintimal angioplasty of a flush occlusion of the superficial femoral artery (SFA) performed via a contralateral common femoral artery approach. Preintervention angiogram of the SFA origin (A). Preintervention angiogram of the reconstituted popliteal artery, just above the knee joint (B). Initiation of the subintimal dissection with creation of the wire loop (C). The wire has re-entered the popliteal artery lumen with the glide microcatheter before expansion (D).

before discharge and 6 months after discharge. Peripheral angiography was performed when there was evidence of a hemodynamically significant stenosis identified by duplex scan.

\section{Definitions}

Primary patency was defined as a treated vessel without a significant restenosis or a need for repeat revascularization. Secondary patency was defined as a target vessel that subsequently became totally occluded and was reopened by repeat revascularization. Technical success was defined as a stenosis less than $30 \%$ on completion angiography and velocity ratio on duplex scan less than 1.5 comparing the treated artery with the proximal patent vessel. Absence of flow determined by

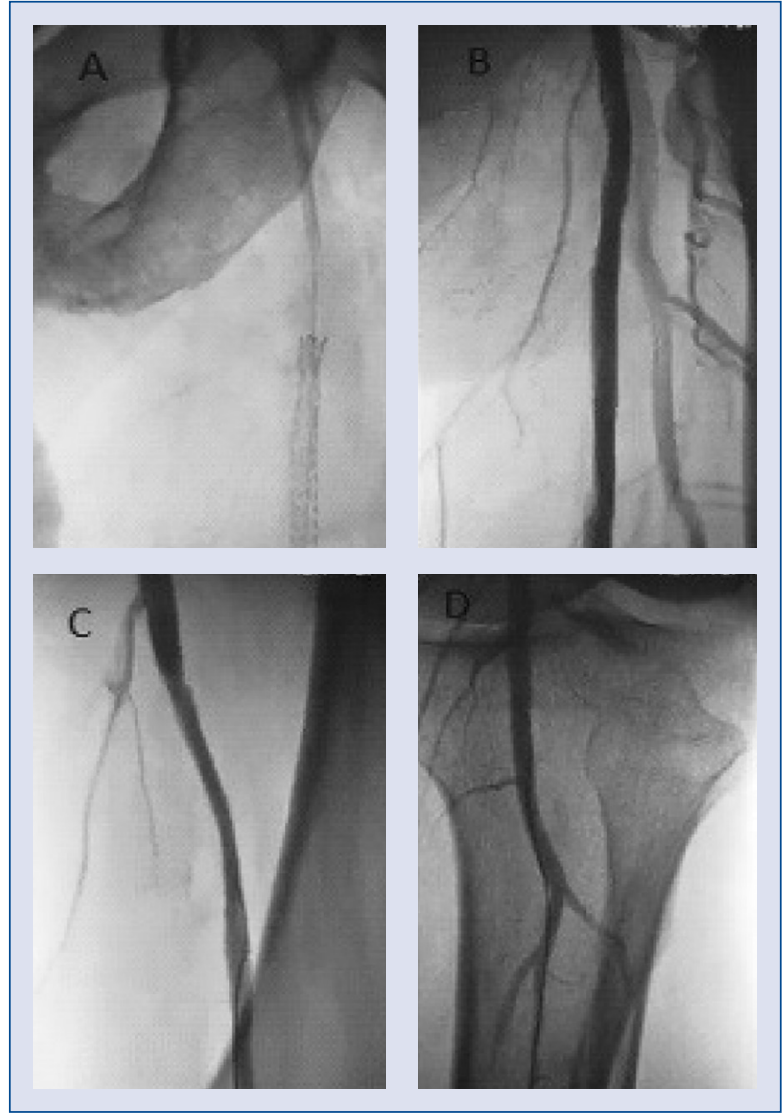

Figure 2. Subintimal angioplasty and stenting of a flush occlusion of the superficial femoral artery (SFA) performed via a contralateral common femoral artery approach (A). Completion angiogram showing patent SFA, popliteal, tibial perineal trunk, posterior tibial, and anterior tibial arteries (B, C, D).

duplex scan indicated occlusion or total restenosis following revascularization.

Systolic velocity greater than $200 \mathrm{~cm} / \mathrm{s}$ and a ratio of greater than 2.5 indicated restenosis of the target vessel. Periprocedural complications included death, acute myocardial infarction, stroke within $48 \mathrm{~h}$ after the procedure, rupture of the target vessel, bleeding which requires blood transfusion, distal embolization, retroperitoneal hematoma, pseudoaneurysm and other access site complications such as groin hematoma.

\section{Statistical analysis}

Quantitative values are expressed as mean \pm \pm standard deviation. The Statistical Package for Social Sciences (SPSS) statistical software package (version 10.0, Inc., Chicago, USA) was used to perform all statistical calculations. 
Table 1. Demographic and clinical characteristics of patients $(n=74)$.

\begin{tabular}{lc}
\hline Variable & N (\%) \\
\hline Age [years] & $65.4 \pm 15.2$ \\
Male gender & $32(43 \%)$ \\
Critical limb ischemia (Rutherford & $16(22 \%)$ \\
categories; 3-6) & \\
Rest pain & $14(19 \%)$ \\
Ulceration & $15(20 \%)$ \\
Gangrene & $8(11 \%)$ \\
Claudication (Rutherford & $21(28 \%)$ \\
categories; 1-3) & \\
Coronary artery disease & $36(49 \%)$ \\
Hyperlipidemia & $32(43 \%)$ \\
Hypertension & $56(76 \%)$ \\
Diabetes mellitus & $48(65 \%)$ \\
Smoking & $54(73 \%)$ \\
Renal insufficiency & $8(11 \%)$ \\
\hline
\end{tabular}

\section{Results}

Seventy four patients with a mean age of $65.4 \pm 15.2$ years underwent SIA plus stenting for chronic total occlusions of femoropopliteal arteries. Patient baseline characteristics showed that $43 \%$ of the participants were male, $73 \%$ were active smokers, $65 \%$ were diabetic, $76 \%$ had hypertension, $49 \%$ had concomitant coronary artery disease and 43\% had hyperlipidemia. According to Rutherford classification [14], 21 (28\%) patients were included in categories 1-3. Patients with ischemic rest pain and limb ulceration consisted of $14(19 \%)$ and $15(20 \%)$ participants, respectively. Eight (11\%) of the patients had gangrene or tissue loss in the study group. Table 1 demonstrates demographic and clinical characteristics of the patients.

The initial procedural success was achieved in 72 of 74 cases $(97 \%)$. SIA and stenting did not succeed in 2 of 74 patients (3\%). All the lesions were revascularized by SIA and stenting in 72 patients.
Table 2. Procedural characteristics of the study population $(n=72)$.

\begin{tabular}{lc}
\hline Variable & $\mathbf{N}(\%)$ \\
\hline Location of SIA and stenting & \\
SFA only & $40(55 \%)$ \\
SFA-popliteal & $30(42 \%)$ \\
Popliteal only & $2(3 \%)$ \\
Number of stents & 156 \\
Stent length & $13.4 \pm 8.2$ \\
Stent diameter & $6.9 \pm 2.6$ \\
Stent overlap & $36(50 \%)$ \\
\hline SIA - subintimal angioplasty: SFA - superficial femoral artery
\end{tabular}

Target lesions were located in the SFA in $55 \%$ of the patients ( 40 of 72 cases). The remaining lesion localizations were $42 \%$ in femoropopliteal and $3 \%$ in popliteal (only) arteries respectively. The mean stent length was $13.4 \pm 8.2 \mathrm{~cm}$ and the mean stent diameter was $6.9 \pm 2.6 \mathrm{~mm}$. In 36 cases $(50 \%$ of the patients), stent overlapping was performed to cover the entire lesion. Table 2 displays procedural characteristics of the study population.

There were no procedure related deaths in the study group. Complications were limited to distal embolus, groin hematoma, femoral pseudoaneurysm, and rupture of SFA-external iliac artery junction. There was no retroperitoneal hematoma observed. Distal embolization leading to acute limb ischemia ( 2 patients, $3 \%$ ) was managed by intra-arterial thrombolysis (alteplase/tissue plasminogen activator, Actilyse) and recanalization was maintained by fibrinolysis and limb salvage was achieved. No blood transfusion or surgical therapy was required in patients with groin hematomas (2 cases, $3 \%$ ). Only $1(1 \%)$ of the patients exhibited femoral pseudoaneurysm which was treated via ultrasound directed thrombin injection. Rupture of the SFA-external iliac artery junction was observed in $1(1 \%)$ patient who was successfully treated by implantation of graft covered stent during the procedure. Table 3 summarizes

Table 3. Clinical conditions complicating the procedures.

\begin{tabular}{lll}
\hline Complications & $\mathbf{N}(\%)$ & Modality of treatment \\
\hline Distal embolus & $2(3 \%)$ & Treated with thrombolysis \\
Groin hematoma & $2(3 \%)$ & Managed by observation \\
Femoral pseudoaneurysm & $1(1 \%)$ & Treated with ultrasound directed thrombin injection \\
Retroperitoneal hematoma & $0(0 \%)$ & \\
Ruptured junction of SFA-external iliac artery & $1(1 \%)$ & Treated with graft-covered stent during procedure \\
\hline
\end{tabular}

SFA - superficial femoral artery 
Table 4. Outcome of femoropopliteal subintimal angioplasty (SIA) and stenting at initial and the $6^{\text {th }}$ month.

\begin{tabular}{lc}
\hline Variable & $\mathbf{N}(\%)$ \\
\hline Successful initial SIA and stenting & $72(97 \%)$ \\
Unsuccessful initial SIA and stenting & $2(3 \%)$ \\
Total restenosis (occlusion) & $1(1 \%)$ \\
at the $6^{\text {th }}$ month & \\
Critical stenosis at the $6^{\text {th }}$ month & $3(4 \%)$ \\
Total amputations & $4(5 \%)$ \\
\hline
\end{tabular}

complications and the applied treatment modalities following the procedures.

The procedural success for SIA and stenting was achieved in majority of patients (72 patients, $97 \%)$. The procedural failure was observed in only $2(3 \%)$ patients. The follow-up period was 6 months for all the patients following femoropopliteal SIA and stenting. At the end of the 6 -month period, $4(5 \%)$ amputations were observed. Only $1(1 \%)$ case displayed total occlusion of the target vessel and $3(4 \%)$ patients exhibited critical stenosis in already revascularized vessels (Table 4).

\section{Discussion}

Chronic total occlusions of femoropopliteal segment account for a significant portion of peripheral vascular lesions which represent challenging technical periprocedural problems that have to be overcome by the interventionalists. PTA of long SFA occlusions with an intraluminal approach usually requires long time consuming procedures, use of excess equipment, and high volume experienced operators. Technical failure of intraluminal angioplasty is mostly attributed to long segment occlusions [15]. In cases of occlusions $>10 \mathrm{~cm}$ long, the primary patency rate of intraluminal angioplasty with stents was $<30 \%$ [16]. Subintimal recanalization with distal re-entry has been successfully used since it has been defined by Bolia et al. [1, 2]. London et al. [17] reported a $90 \%$ technical success rate for SIA of SFA in a series of patients with a mean occlusion length of $15 \mathrm{~cm}$. Recently, Soga et al. [18] have published the initial and 3-year results of 902 patients undergoing SIA vs. intraluminal angioplasty with stenting in long femoropopliteal occlusions and declared that patency rates were similar in either approaches. The investigators of this study also reported longer procedure time and high crossover rates in intraluminal approach group, so SIA with stenting was suggested to be preferred in the treatment of long femoropopliteal occlusions [18]. SIA is performed by creating an extraluminal channel between intimal and medial layers of the artery which contains little atherosclerotic plaque or thrombus. The relatively low content of thrombus or plaque burden also makes SIA more advantageous than the intraluminal approach. Previous studies reveal high technical success rates ranging from $74 \%$ to $92 \%$ with few complications [19]. The most technical failures reported previously were due to failure to pass wire into the extraluminal space or re-entry into the true lumen. We experienced a high technical success rate $(97 \%)$ and the remaining unsuccessful cases were mainly associated with extreme calcification that prevents re-entry into the distal true lumen. The complications reported in previous reports were perforation, vessel rupture, distal embolization and occlusion of collaterals mostly without a need for surgical repair $[20,21]$. We have experienced 1 (1\%) vessel rupture which was treated by implantation of a covered stent. Distal embolization occurred in $2(3 \%)$ cases and was managed by catheter directed thrombolysis. A bolus of $1 \mathrm{mg}$ tissue plasminogen activator (t-PA) was given into the infusion catheter and $1 \mathrm{mg} / \mathrm{h}$ t-PA was administered for $12 \mathrm{~h}$ in cases of acute distal embolization. The next day following thrombolysis, an angiography was performed to see the final flow in the artery.

Several studies have been conducted on the endovascular treatment of SFA occlusions $>10 \mathrm{~cm}$ in length and angioplasty alone was used achieving a mean primary patency rate of $46 \%$ at 12 months [22-24]. Other studies have reported the results of bail-out stenting strategies (stenting performed if only angioplasty alone fails) which reveal a mean primary patency rate of 55\% at 12 months [25-28].

A meta-analysis by Muradin et al. [29] declared that there is no substantial difference between angioplasty alone and stenting in terms of long-term patency rate, whereas in the treatment of long SFA occlusions, stenting is superior to angioplasty to gain better patency outcomes. The use of stents in SFA has lowered the incidence of early stenoses which is mostly due to elastic recoil, residual lesion, and flow limiting dissections even in calcified complex arteries [30]. However, despite these results, routine stenting of SFA occlusions remains controversial because of the development of intimal hyperplasia leading to in-stent restenosis which dominantly occurs in long lesions or in multiple stents deployed [31]. Boufi et al. [32] have recently presented the long term 
results of SIA plus stenting in a homogenous series of claudicants with femoropopliteal occlusions and declared that SIA plus stenting is an effective and useful option for the management of claudicants with femoropopliteal occlusions which may be considered complementary to surgical bypass. Our findings are partly consistent with this study which indicates stenting combined with SIA as a valuable revascularization strategy for these long diffuse occlusions with the use of new stents which are more durable against mechanic forces. We have to point out that the follow-up period should be longer to see the late term outcomes in terms of restenosis or re-occlusions as a study limitation.

\section{Conclusions}

Percutaneous SIA and stenting of chronic long femoropopliteal occlusions show good initial and mid-term patency rates. Complication rates are also favorable compared with other revascularization options such as surgery or percutaneous luminal angioplasty with or without stenting.

\section{Conflict of interest: None declared}

\section{References}

1. Bolia A, Brennan J, Bell PR. Recanalisation of femoropopliteal occlusions: improving success rate by subintimal recanalisation. Clin Radiol, 1989; 40: 325.

2. Bolia A, Bell PR. Femoropopliteal and crural artery recanalization using subintimal angioplasty. Semin Vasc Surg, 1995; 8: 253-264.

3. Desgranges P, Boufi M, Lapeyre M et al. Subintimal angioplasty: feasible and durable. Eur J Vasc Endovasc Surg, 2004; 28: 138-141.

4. Scott EC, Biuckians A, Light RE et al. Subintimal angioplasty for the treatment of claudication and critical limb ischemia: 3-year results. J Vasc Surg, 2007; 46: 959-964.

5. Flørenes T, Bay D, Sandbaek G et al. Subintimal angioplasty in the treatment of patients with intermittent claudication: Long term results. Eur J Vasc Endovasc Surg, 2004; 28: 645-650.

6. Laxdal E, Jenssen GL, Pedersen G, Aune S. Subintimal angioplasty as a treatment of femoropopliteal artery occlusions. Eur J Vasc Endovasc Surg, 2003; 25: 578-582.

7. Met R, Van Lienden KP, Koelemay MJ, Bipat S, Legemate DA, Reekers JA. Subintimal angioplasty for peripheral arterial occlusive disease: a systematic review. Cardiovasc Intervent Radiol, 2008; 31: 687-697.

8. Bown MJ, Bolia A, Sutton AJ. Subintimal angioplasty: metaanalytical evidence of clinical utility. Eur J Vasc Endovasc Surg, 2009; 38: 323-337.

9. Keeling AN, Khalidi K, Leong S et al. Subintimal angioplasty: Predictors of longterm success. J Vasc Interv Radiol, 2009; 20: 1013-1022.

10. Markose G, Miller FN, Bolia A. Subintimal angioplasty for femoro-popliteal occlusive disease. J Vasc Surg, 2010; 52: 1410-1416.

11. Keeling AN, Khalidi K, Leong S et al. Subintimal angioplasty: Predictors of long-term success. J Vasc Interv Radiol, 2009; 20: 1013-1022.

12. Kim SJ, Kim W, Kim JB, Hong MJ, Kang WY, Hwang SH. Determinants of procedural success and patency following subintimal angioplasty in patients with TASC C and D femoropopliteal arterial disease. Circ J, 2010; 74: 1959-1964.

13. Yilmaz S, Sindel T, Ceken K, Alimoglu E, Lüleci E. Subintimal recanalization of long superficial femoral artery occlusions through the retrograde popliteal approach. Cardiovasc Intervent Radiol, 2001; 24: 154-160.

14. Rutherford RB, Baker JD, Ernst C et al. Recommended standards for reports dealing with lower extremity ischemia: revised version. J Vasc Surg, 1997; 26: 517-538.

15. Capek P, McLean GK, Berkowitz HD. Femoropopliteal angioplasty. Factors influencing long-term success. Circulation, 1991; 83: $170-180$.

16. Gray BH, Olin JW. Limitations of percutaneous transluminal angioplasty with stenting for femoropopliteal arterial occlusive disease. Semin Vase Surg, 1997; 10: 8-16.

17. London NJ, Srinivasan R, Naylor AR et al. Subintimal angioplasty of femoropopliteal artery occlusions: The long-term results. Eur J Vasc Surg, 1994; 8: 148-155.

18. Soga $Y$, Lida O, Suzuki K et al. Initial and 3-year results after subintimal versus intraluminal approach for long femoralpopliteal occlusion treated with a self-expandable nitinol stent. J Vasc Surg, 2013; 58: 1547-1555.

19. Ko YK, Kim JS, Choi DH, Jang Y, Shim WH. Improved technical success and midterm patency with subintimal angioplasty compared to intraluminal angioplasty in long femoropopliteal occlusions. J Endovasc Ther, 2007; 14: 374-373.

20. Lipsitz EC, Ohki T, Veith FJ et al. Fate of collateral vessels following subintimal angioplasty. J Endovase Ther, 2004; 11:269-273.

21. Lipsitz EC, Veith FJ, Ohki T. The value of subintimal angioplasty in the management of critical lower extremity ischemia: Failure is not always associated with a rethreatened limb. J Cardiovase Surg, 2004; 45: 231-237.

22. Jamsen TS, Manninen HI, Jaakkola PA et al. Long-term outcome of patients with claudication after balloon angioplasty of the femoropopliteal arteries. Radiology, 2002; 225: 345-352.

23. Laxdal E, Jenssen GL, Pedersen G et al. Subintimal angioplasty as a treatment of femoropopliteal artery occlusions. Eur J Vasc Endovasc Surg, 2003; 25: 578-582.

24. Lofberg AM, Karacagil S, Ljungman C et al. Percutaneous transluminal angioplasty of the femoropopliteal arteries with chronic critical lower limb ischemia. Vasc Surg, 2001; 34: 114-121.

25. Cheng SW, Ting AC, Wong J. Endovascular stenting of superficial femoral artery stenosis and occlusions: Results and risk factor analysis. Cardiovasc Surg, 2001; 9: 133-140.

26. Conroy RM, Gordon IL, Tobis JM et al. Angioplasty and stent placement in chronic occlusion of the superficial femoral artery: technique and results.Vasc Interv Radiol, 2001; 11: 1009-1020 .

27. Jahnke T, Voshage G, Muller-Hulsbeck S et al. Endovascular placement of self-expanding nitinol stents for the treatment of femoropopliteal obstructive disease. J Vasc Interv Radiol, 2002; 13: 257-266.

28. Surowiec SM, Davies MG, Eberly SW et al. Percutaneous angioplasty and stenting of the superficial femoral artery. J Vasc Surg, 2005; 41: 269-278.

29. Muradin GS, Bosch JL, Stijnen T et al. Balloon dilation and stent implantation for treatment of femoropopliteal arterial disease: Metaanalysis. Radiology, 2001; 221: 137-145.

30. Schillinger M, Minar E. Endovascular stent implantation for treatment of peripheral artery disease. Eur J Clin Invest, 2007; 37: 165-170.

31. Tsetis D, Belli AM . Guidelines for stenting in infrainguinal arterial disease. Cardiovasc Intervent Radiol, 2004; 27: 198-203.

32. Boufi M, Azghari A, Belahda K, Loundou AD, Hartung Oa, Alimi YS. Subintimal recanalization plus stenting or bypass for management of claudicants with femoropopliteal occlusions. Eur J Vasc Endovasc Surg, 2013; 46: 347-352. 